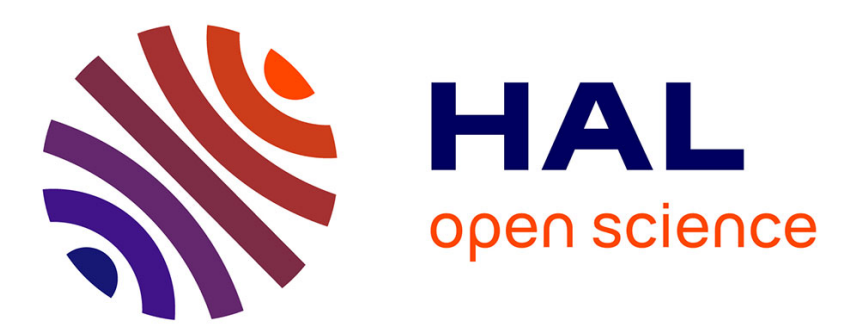

\title{
Spatial Factors for Triplet Fusion Reaction of Singlet Oxygen Photosensitization
}

\author{
Shuming Bai, Mario Barbatti
}

\section{To cite this version:}

Shuming Bai, Mario Barbatti. Spatial Factors for Triplet Fusion Reaction of Singlet Oxygen Photosensitization. Journal of Physical Chemistry Letters, 2017, 8 (21), pp.5456-5460. 10.1021/acs.jpclett.7b02574 . hal-02288769

\section{HAL Id: hal-02288769 \\ https://hal-amu.archives-ouvertes.fr/hal-02288769}

Submitted on 16 Sep 2019

HAL is a multi-disciplinary open access archive for the deposit and dissemination of scientific research documents, whether they are published or not. The documents may come from teaching and research institutions in France or abroad, or from public or private research centers.
L'archive ouverte pluridisciplinaire HAL, est destinée au dépôt et à la diffusion de documents scientifiques de niveau recherche, publiés ou non, émanant des établissements d'enseignement et de recherche français ou étrangers, des laboratoires publics ou privés. 


\title{
Spatial Factors for Triplet Fusion Reaction of
}

\section{Singlet Oxygen Photosensitization}

\author{
Shuming Bai* and Mario Barbatti*
}

Aix Marseille Univ, CNRS, ICR, Marseille, France.

First-principles quantum-chemical description of photosensitized singlet oxygen generation kinetics is challenging due to the intrinsic complexity of the underlying triplet fusion process in a floppy molecular complex with open-shell character. With a quantum-chemical kinetic model specifically tailored to deal with this problem, the reaction rates are investigated as a function of intermolecular incidence direction, orientation, and distance between $\mathrm{O}_{2}$ and the photosensitizer. The adopted photosensitizer, 6-azo-2-thiothymine, combines practical interest and prototypical variability. The study quantitatively determined maximum singlet oxygen generation rates for fifteen incidence/orientation directions, showing that they span five orders of magnitude between the largest and the smallest rate. Such information may provide a hands-on guideline for the experimental molecular design of new photosensitizers as well as further higher-level theoretical research.

\section{TOC graph}

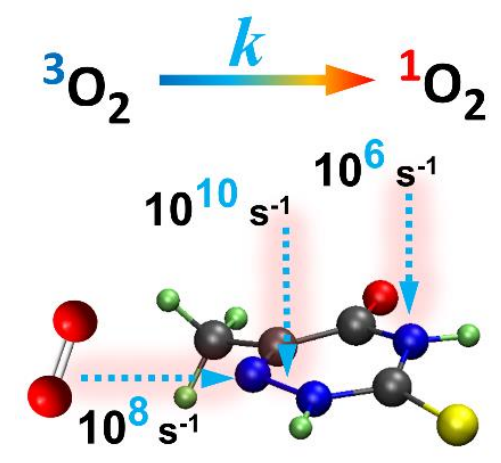

Keywords: triplet fusion, singlet oxygen, photosensitization, kinetic model 
The investigation of singlet oxygen — including its generation, reactivity properties, and physiological cytotoxicity — has been a popular subject for several decades because of its broad importance for chemical synthesis, environmental sciences, phototherapy, and physiology. ${ }^{1-3}$ In many applications, the extra energy needed to generate the singlet species from the triplet $\mathrm{O}_{2}$ ground state is harvested from the excited states of a photosensitizer (PS). ${ }^{4-5}$ During this photosensitization process, singlet oxygen is generated through the spin-conserving reaction

$$
{ }^{1}\left[{ }^{3} P S+{ }^{3} O_{2}\right] \longrightarrow{ }^{1}\left[{ }^{1} P S+{ }^{1} O_{2}\right],
$$

which is dominant in solution. ${ }^{6}$ Various types of photosensitizers have been developed aiming at specific applications. ${ }^{7-8}$ Among them, several thiopyrimidine derivatives have been recently reported as effective, high ${ }^{1} \mathrm{O}_{2}$-yield phototherapeutic agents. ${ }^{4,9-10}$ Curiously, the same thiopyrimidines have also been reported as carcinogenic agents due to singlet oxygen generation as a side-effect of immunotherapy. ${ }^{11-13}$

Theoretical and computational chemistry, including quantum chemical calculations and reaction kinetics, has continuously contributed to this topic. ${ }^{1,14-15}$ It has offered essential assistance to experimental studies, rationalizing the empirical relationship between rates and properties of photosensitizers, ${ }^{16}$ predicting possible reaction mechanisms based on the direct quantum chemical calculation of the excited states of the PS-O $\mathrm{O}_{2}$ complex, ${ }^{1,17}$ and even delivering preliminary estimates of the reaction kinetics. ${ }^{18}$

Nevertheless, research based on first-principles quantum-chemical calculations of the photosensitization process is still scarce. ${ }^{18}$ Theoretical investigations of isolated PS, including its photoexcitation and decay dynamics to the triplet state, have been the focus in the past years. ${ }^{9,}{ }^{19-25}$ Recently, we investigated the intrinsic decay of the 6-aza-2-thiothymine (6n-2tThy) triplet state, a process competing with the singlet oxygen generation. ${ }^{26}$ A compelling reason to investigate this system, whose structure is shown in Figure 1(a), is that it is the simplest known thiopyrimidine with high ${ }^{1} \mathrm{O}_{2}$ quantum yield $(\Phi=0.69) .{ }^{27}$ After determining how the triplet PS may decay without forming ${ }^{1} \mathrm{O}_{2}$, the study of Reaction (1) becomes 
the next target. In fact, we have already taken the first step by developing a kinetic model to explore this type of triplet fusion (or triplet annihilation) reaction quantitatively. ${ }^{28}$ The model, named Divide-to-Conquer (DtC), is also the basis for the present work.
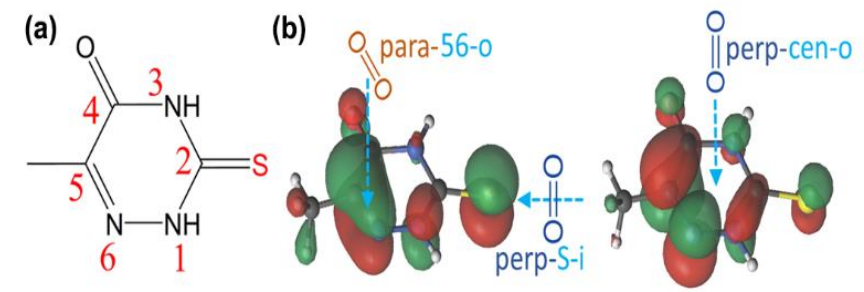

Figure 1. (a) Structure and numbering of 6n-2tThy; (b) Singly-occupied orbitals of the $\mathrm{T}_{1}$ state of 6n-2tThy. Three different incidence/orientation directions are indicated in (b).

Various strategies have been developed to charge and energy transfer reaction rates. ${ }^{28-35}$ In this work, we adopt the DtC model, which allows calculating the rate of a triplet fusion reaction in a floppy system is given in terms of an extended Marcus model. In addition to the conventional coordinate $\mathbf{R}$ defining the Marcus parabolic free energies, a coordinate D-defining the intermolecular orientation, direction, and distance between PS and $\mathrm{O}_{2}$-is considered as well. For each $\mathbf{D}$, the rate $k_{i j}$ between states $i$ and $j$ is determined by the quasi-Marcus formula:

$$
k_{i j}=\frac{2 \pi}{\hbar}\left|V_{\text {coup }}\right|^{2} \frac{1}{\sqrt{4 \pi \lambda k_{B} T}} \exp \left(-\frac{\Delta G^{\ddagger}}{k_{B} T}\right) .
$$

To calculate Eq. (2) requires three quantities: (1) the diabatic coupling $V_{\text {coup }},(2)$ the activation free energy $\Delta G^{\ddagger}$, and (3) the reorganization energy $\lambda$. With a reasonable assumption that the interaction between the monomers mainly depends on the intermolecular vector $\mathbf{D}$, the calculation of these three quantities is simplified into a practical level. The model is further approximated by neglecting entropic effects and kinetic contributions to enthalpic effects, which allows replacing $\Delta G^{\ddagger}$ by the activation energy $\Delta E^{\ddagger}$ in 
Eq. (2). $V_{\text {coup }}$ is computed by integration of nonadiabatic couplings along one-dimensional paths. The model is presented in more details in the Supporting Information SI-1. Technical details on the DtC model have been described in Ref. ${ }^{28}$.

Because of the floppy structure in solution, it is hard to predict which orientation between PS and $\mathrm{O}_{2}$ holds the highest reaction probability or to determine a single structure that could be representative enough to provide a nearly complete picture. Thus, to study the impact of the incidence direction, orientation, and distance between PS and $\mathrm{O}_{2}$ — variables that we call spatial factors-becomes inescapable. Although researchers such as Ogilby and coauthors have already realized the importance of orientation and conformation for organic-oxygen complexes ${ }^{1}$ and used it for spectroscopic interpretation, ${ }^{36-37}$ this topic is still underdeveloped when dealing with photosensitization. In a previous work by Serrano-Pérez and co-workers, these spatial factors were considered, taking ethylene as a model for the PS and using empirical density of states. ${ }^{18}$ In this work, we employ the DtC model to deliver a first-principles investigation of the spatial factors for a realistic PS, ${ }^{28}$ systematically determining the reaction rates for fifteen incidence/orientation directions, spanning the whole volume around the PS.

Throughout the paper, each of the incidence/orientation directions is labeled as $<$ orientation $>$ - $<$ target $>$ $<$ placement $>$. The label $<$ orientation $>$ refers to the orientation of the $\mathrm{O}_{2}$ axis in relation to the ring plane of $6 \mathrm{n}-2 \mathrm{tThy}$, and may take para or perp values for parallel or perpendicular. $<$ target $>$ refers to the group of atoms in $6 n-2 t$ Thy that is approached by $\mathrm{O}_{2} .<$ placement $>$ indicates whether $\mathrm{O}_{2}$ rests out of the ring plane $(o)$ or in the ring plane $(i)$. Three examples of these incidence/orientation directions are illustrated in Figure 1(b). For instance, the label para-56-o means that an $\mathrm{O}_{2}$ parallel to the ring plane approaches 6n-2tThy from out of plane towards atoms C5 and N6. Detailed information about each direction is provided in the SI-3. 
For each of the fifteen directions, we computed $\Delta E^{\ddagger}, V_{\text {coup }}$, and $\lambda$ based on results obtained at the multi-state complete active space perturbation theory to the second order (MS-CASPT2) and complete active state self-consistent field (CASSCF) levels (See SI-2 for computational details and SI-4 for Cartesian coordinates). A typical result for one of the directions (perp-56-o) is shown in Figure 2(a) (for the other directions, the results are given in the SI-3). With this information, the rates to generate ${ }^{1} \mathrm{O}_{2}$ in the different singlet states are calculated as a function of distance using Eq. (2) (see Figure 2(b) for a particular case).
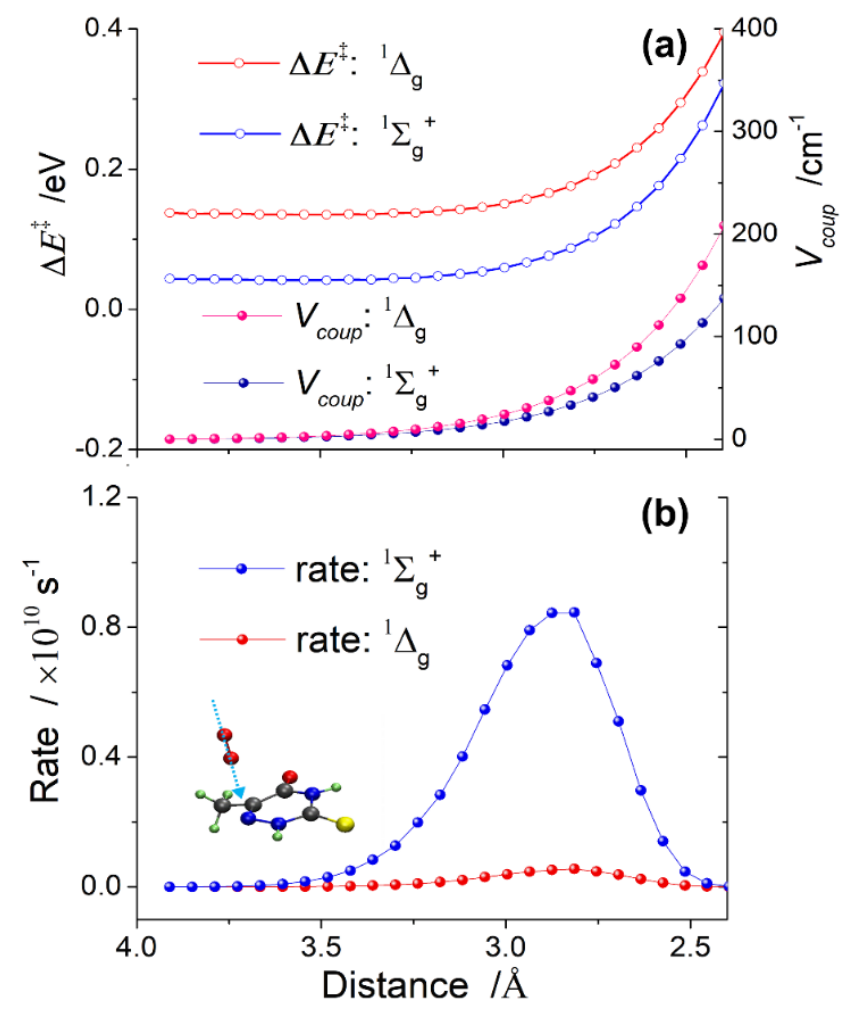

Figure 2. Results for perp-56-o direction as a function of distance: (a) activation energy $\Delta E^{\ddagger}$ and diabatic coupling $V_{\text {coup }}$; (b) singlet oxygen generation rate. (For all other directions, see SI-3.)

Because the $S_{0}^{P S-O_{2}}$ and $S_{1}^{P S-O_{2}}$ states of the complex are nearly degenerated, both corresponding to the configurations of the ${ }^{1} \Delta_{g}$ oxygen molecule with PS in its ground state $S_{0}^{P S}$, we treated them as a single 
state by combining their diabatic couplings into a single quantity $V_{\text {coup }}\left({ }^{1} \Delta_{g}\right)=\sqrt{V_{\text {coup }}\left({ }^{1} \Delta_{g 1}\right)^{2}+V_{\text {coup }}\left({ }^{1} \Delta_{g 2}\right)^{2}}$. In the case of the $S_{2}^{P S-O_{2}}$ state, $\mathrm{O}_{2}$ is in the ${ }^{1} \Sigma_{g}^{+}$configuration with PS still in $S_{0}^{P S}$. In the $S_{3}^{P S-O_{2}}$ state, $\mathrm{O}_{2}$ is in the ${ }^{3} \Delta_{\mathrm{g}}$ configuration, while PS is in the $T_{1}^{P S}$ state. This state order is kept independently of the direction, orientation, and distance. (It may change for very short distances not reached under normal conditions.) Thus, the reaction from the complex state $S_{3}^{P S-O_{2}}$ to $S_{0}^{P S-O_{2}}$ or $S_{1}^{P S-O_{2}}$ always generates ${ }^{1} \Delta_{\mathrm{g}}$ singlet oxygen, while the reaction to $S_{2}^{P S-O_{2}}$ generates ${ }^{1} \Sigma_{g}^{+}$singlet oxygen.

Table 1. Calculated maximum rates for generation of singlet oxygen in the ${ }^{1} \Sigma_{g}^{+}$and ${ }^{1} \Delta_{g}$ states through Reaction (1) for different orientations and directions.

\begin{tabular}{|c|c|c|c|c|c|c|c|c|}
\hline $\begin{array}{l}\text { Path } \\
\text { label }\end{array}$ & $\begin{array}{l}D_{\max } \\
/ \AA\end{array}$ & $\begin{array}{l}\Delta E^{\ddagger}\left({ }^{1} \Sigma_{g}^{+}\right) \\
/ \mathrm{eV}\end{array}$ & $\begin{array}{l}\Delta E^{\ddagger}\left({ }^{1} \Delta_{g}\right) \\
/ \mathrm{eV}\end{array}$ & $\begin{array}{l}V_{\text {coup }}\left({ }^{1} \Sigma_{g}^{+}\right) \\
/ \mathrm{cm}^{-1}\end{array}$ & $\begin{array}{l}V_{\text {coup }}\left({ }^{1} \Delta_{g}\right) \\
/ \mathrm{cm}^{-1}\end{array}$ & $\begin{array}{l}k\left({ }^{1} \Sigma_{g}^{+}\right) \\
/ 10^{10} \mathrm{~s}^{-1}\end{array}$ & $\begin{array}{l}k\left({ }^{1} \Delta_{g}\right) \\
/ 10^{10} \mathrm{~s}^{-1}\end{array}$ & $\begin{array}{l}{ }^{1} \Sigma_{g}^{+} I^{1} \Delta_{g} \\
k \text { ratio }\end{array}$ \\
\hline para-61-o & 2.90 & 0.0814 & 0.171 & 47.5 & 209 & 2.15 & 1.28 & 1.68 \\
\hline perp-61-o & 2.87 & 0.0821 & 0.175 & 32.6 & 77.6 & 0.992 & 0.154 & 6.44 \\
\hline para-56-o & 2.80 & 0.0776 & 0.179 & 38.5 & 136 & 1.64 & 0.402 & 4.08 \\
\hline perp-56-o & 2.81 & 0.0872 & 0.176 & 33.3 & 47.2 & 0.845 & 0.055 & 15.4 \\
\hline para-45-o & 3.00 & 0.0878 & 0.180 & 24.1 & 65.7 & 0.432 & 0.0908 & 4.76 \\
\hline perp-45-o & 2.93 & 0.0983 & 0.192 & 8.00 & 10.4 & 0.0318 & $1.41 \mathrm{E}-3$ & 22.6 \\
\hline perp-cent-o & 2.99 & 0.0829 & 0.176 & 21.1 & 41.5 & 0.402 & 0.0430 & 9.35 \\
\hline para-cent-o & 3.12 & 0.110 & 0.167 & 3.61 & 89.8 & $0.408 \mathrm{E}-2$ & 0.282 & $1.44 \mathrm{E}-2$ \\
\hline perp-5-o & 2.94 & 0.0845 & 0.178 & 12.1 & 18.9 & 0.124 & $0.822 \mathrm{E}-2$ & 15.1 \\
\hline perp-6-i & 2.99 & 0.0693 & 0.171 & 4.89 & 39.8 & 0.0364 & 0.0478 & 0.762 \\
\hline para-6-i & 2.83 & 0.0855 & 0.194 & 0.176 & 7.15 & $0.251 \mathrm{E}-4$ & $0.621 \mathrm{E}-3$ & $4.04 \mathrm{E}-2$ \\
\hline para-S-i & 2.99 & 0.0974 & 0.191 & 0.516 & 6.93 & $0.136 \mathrm{E}-3$ & $0.666 \mathrm{E}-3$ & 0.204 \\
\hline
\end{tabular}




\begin{tabular}{lllllllll} 
perp-S-i & 3.14 & 0.112 & 0.184 & 0.116 & 31.8 & $0.393 \mathrm{E}-5$ & 0.0185 & $2.12 \mathrm{E}-4$ \\
perp-3-o & 3.03 & 0.0648 & 0.174 & 0.612 & 1.56 & $0.680 \mathrm{E}-3$ & $0.656 \mathrm{E}-4$ & 10.4 \\
para-3H-i & 2.15 & 0.0683 & 0.154 & 0.195 & 0.289 & $0.606 \mathrm{E}-4$ & $0.478 \mathrm{E}-5$ & 12.7 \\
\hline
\end{tabular}

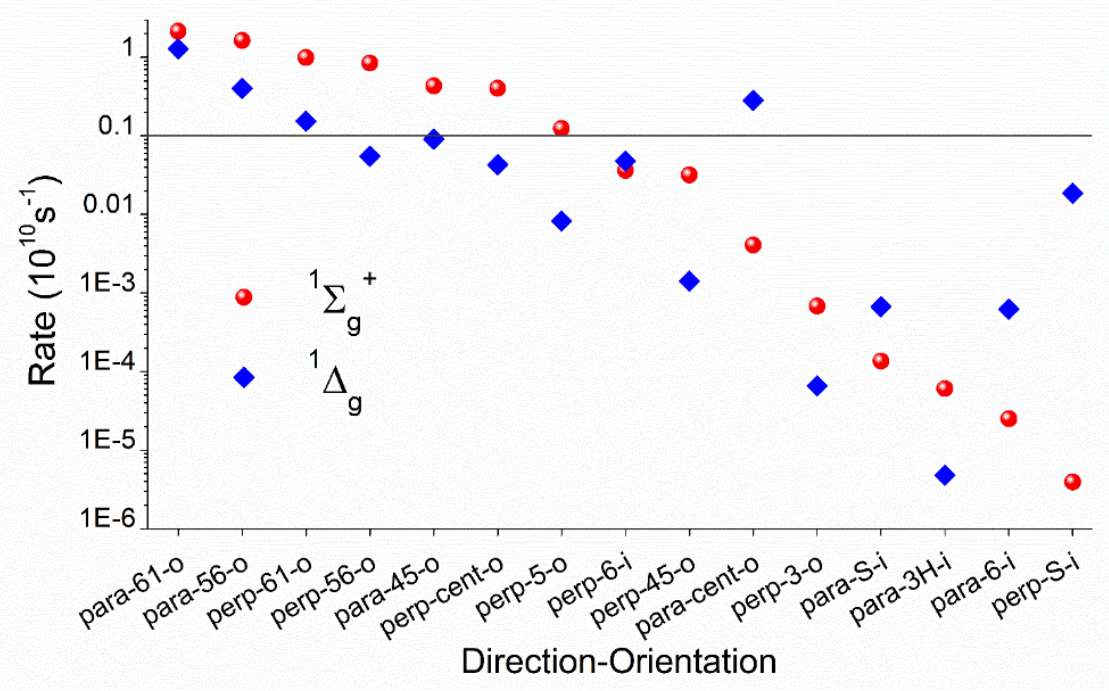

Figure 3. Maximum calculated rates for ${ }^{1} \Sigma_{g}^{+}$(red circles) and ${ }^{1} \Delta_{g}$ (blue lozenges) singlet oxygen generation for different directions.

The main results for all fifteen directions are summarized in Table 1 . They are collected for the distance that maximizes the rate in each case. Based on these values, we will proceed with the discussion of the spatial factors. The singlet oxygen generation rate is the most significant result, so we plot it in Figure 3.

The rates strongly depend on the direction, spanning five orders of magnitude between the largest $\left(2.15 \times 10^{10} \mathrm{~s}^{-1}\right)$ and the lowest $\left(0.4 \times 10^{5} \mathrm{~s}^{-1}\right)$. For all rates larger than $0.1 \times 10^{10} \mathrm{~s}^{-1}$, the directions are out-ofplane (-o), having atoms C4/C5/N6 as targets, exactly where the highest spin density of $\mathrm{T}_{1}$ peaks (see the single occupied orbitals in Figure 1(b)). This finding implies that orbital overlap is a necessary condition for effective photosensitization and indicates the relative importance of different paths to research. For 
6n-2tThy, this happens for an $\mathrm{O}_{2}$ attack from above (or below) the aromatic ring. $\mathrm{O}_{2}$ attack on the ring plane (-i) renders low rates.

Compared with the direction factor, the orientation factor has a much smaller effect on the rate. For the most important directions, ${ }^{1} \Sigma_{g}^{+}$rates for para are about twice those for perp. For ${ }^{1} \Delta_{g}$ rates, para is about 8 times larger than perp. The bigger rates for para orientation are mainly due to stronger diabatic coupling caused by the larger orbital overlap of the degenerated $\pi_{\mathrm{x}} / \pi_{\mathrm{y}}$ of $\mathrm{O}_{2}$ with the singly occupied orbitals of PS for out-of-plane placements, in comparison to the overlap between $\pi_{\mathrm{z}}$ and PS. If the $\mathrm{O}_{2}$ rotation were taken into account, we could expect intermediary rate values between those for para and perp.

For the most productive directions, rates for forming ${ }^{1} \Sigma_{g}^{+}$are larger than to form ${ }^{1} \Delta_{g}$ by 10 to $60 \%$. This means that the direct formation of the final singlet oxygen product $\left({ }^{1} \Delta_{g}\right)$ should occur mainly via internal conversion from ${ }^{1} \Sigma_{g}^{+}$, but still with significant amounts of direct formation. Although experimental ${ }^{1} \Sigma_{g}^{+}{ }^{1} \Delta_{g}$ ratio for 6 n-2tThy is not available, experimental data for more than 40 sensitizers confirm that it is reasonable to expect a ratio superior to the unity. ${ }^{38}$

With a single exception (perp-center-o), all out-of-plane (-o) directions render a ${ }^{1} \Sigma_{g}^{+} I^{1} \Delta_{g}$ ratio larger than the unity (Table 1). This is the opposite from the in-plane (-i) directions, whose ratio is always smaller than the unity. Because the ${ }^{1} \Sigma_{g}^{+}$state lies above the ${ }^{1} \Delta_{g}$ states, the activation energies to populate the former are smaller, which favors larger ${ }^{1} \Sigma_{g}^{+}$rates (as in the -o directions). Nevertheless, when the diabatic coupling is significantly weak (as in the $-\mathrm{i}$ directions), the ${ }^{1} \Delta_{g}$ rate dominates. Therefore, the ratio between ${ }^{1} \Sigma_{g}^{+}$and ${ }^{1} \Delta_{g}$ rates is determined by an interplay between activation energy and diabatic coupling. 
To illustrate the relationship between the final rate and the two key parameters - activation energy and diabatic coupling, we collected the results from Table 1 into Figure 4 . It makes clear that the rates for ${ }^{1} \Sigma_{g}^{+}$ and ${ }^{1} \Delta_{g}$ formation fall into two separate zones of the $\Delta E^{\ddagger} \times V_{\text {coup }}$ space, which implies different mechanisms underlying them. For the same direction, ${ }^{1} \Sigma_{g}^{+}$rates have smaller couplings $\left(V_{\text {coup }}<50 \mathrm{~cm}^{-1}\right)$ than ${ }^{1} \Delta_{g}$, whose coupling may reach $200 \mathrm{~cm}^{-1}$. These smaller couplings are, however, compensated by also smaller activation energies, clustering around $0.08 \mathrm{eV}$ for ${ }^{1} \Sigma_{g}^{+}$and $0.18 \mathrm{eV}$ for ${ }^{1} \Delta_{g}$. As pointed out in previous works, ${ }^{5,39-41}$ the coupling strength is generally associated with a mixing to a charge transfer state. To understand, however, why the couplings are larger in the transfer to ${ }^{1} \Delta_{g}$ than in that to ${ }^{1} \Sigma_{g}^{+}$ requires a more in-depth analysis of the wave functions, which goes beyond the scope of this letter.

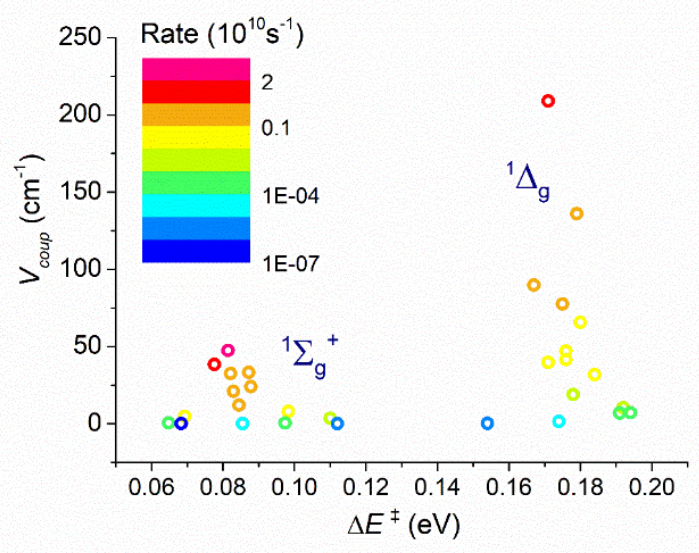

Figure 4. Maximum reaction rates to form ${ }^{1} \Sigma_{g}^{+}$and ${ }^{1} \Delta_{g} \mathrm{O}_{2}$ as a function of activation energy and diabatic coupling for all incidence/orientation directions.

To summarize, we calculated reaction rates for singlet oxygen generation through photosensitization in a PS-O $\mathrm{O}_{2}$ system, using a kinetic model designed to deal with energy transfer in weakly-coupled floppy complexes. Aiming at a conceptual understanding of the importance of the intermolecular orientation, 
direction, and distance for the reaction, we computed the rates for a large number of $\mathrm{PS}-\mathrm{O}_{2}$ configurations, spanning 15 directions and orientations, with distances ranging from 2.5 to $4 \AA$. To cope with the costs of these hundreds of rate determination, quantum chemical level was tuned to a moderate accuracy. This strategy allows determining the most relevant $\mathrm{PS}-\mathrm{O}_{2}$ conformations, which may then be target of further higher-level investigations.

Taking 6-aza-2-thiothymine as PS, the investigation of the spatial factors showed that $\mathrm{O}_{2}$ incidence from above (and parallel to) the ring plane near N1-N6 and C5-N6 bonds (the PS region with highest spin density) maximizes the rates for both ${ }^{1} \Sigma_{g}^{+}$and ${ }^{1} \Delta_{g}$ singlet oxygen. It also showed that in-plane PS-O $\mathrm{O}_{2}$ interaction has vanishing rates; that ${ }^{1} \Sigma_{g}^{+}$formation is the dominant process; and that ${ }^{1} \Sigma_{g}^{+}$and ${ }^{1} \Delta_{g}$ formations have different underlying mechanisms.

The methodology employed here is not restricted to singlet oxygen generation, and it may be applied to other energy transfer reactions, especially to investigate triplet fusion and singlet fission in floppy complexes. To be able to determine rates as a function of intermolecular geometry should contribute to the synergy between experimental and theoretical research on molecular design, as this type of information can directly tell which kind of substituents on specific positions to control reaction yields.

\title{
ASSOCIATED CONTENT
}

\section{Supporting Information.}

Introduction to the $\mathrm{DtC}$ model, details on quantum chemical calculation, results for other directions, Cartesian coordinates. This material is available free of charge via the Internet at http://pubs.acs.org.

\author{
AUTHOR INFORMATION
}

\section{* Corresponding Authors}


E-mail: mario.barbatti@univ-amu.fr; Website: www.barbatti.org

\section{ACKNOWLEDGMENT}

This work was supported by Excellence Initiative of Aix-Marseille University (A*MIDEX) and the project Equip@Meso (ANR-10-EQPX-29-01), both funded by the French Government "Investissements d'Avenir" program. The authors also acknowledge funding from HPC resources from GENCI-CINES (Grant 2017-A0010810012).

\section{REFERENCES}

1. Paterson, M. J.; Christiansen, O.; Jensen, F.; Ogilby, P. R. Invited review - Overview of theoretical and computational methods applied to the oxygen-organic molecule photosystem. Photochem. Photobiol. 2006, 82 (5), 1136-1160.

2. Torring, T.; Helmig, S.; Ogilby, P. R.; Gothelf, K. V. Singlet Oxygen in DNA Nanotechnology. Acc. Chem. Res. 2014, 47 (6), 1799-1806.

3. Ghogare, A. A.; Greer, A. Using Singlet Oxygen to Synthesize Natural Products and Drugs. Chem. Rev. 2016, 116 (17), 9994-10034.

4. Pollum, M.; Jockusch, S.; Crespo-Hernandez, C. E. 2,4-Dithiothymine as a Potent UVA Chemotherapeutic Agent. J. Am. Chem. Soc. 2014, 136 (52), 17930-17933.

5. Schweitzer, C.; Schmidt, R. Physical Mechanisms of Generation and Deactivation of Singlet Oxygen. Chem. Rev. 2003, 103, 1685-1757.

6. Schmidt, R. The Effect of Solvent Polarity on the Balance between Charge Transfer and NonCharge Transfer Pathways in the Sensitization of Singlet Oxygen by $\pi \pi^{*}$ Triplet States. J. Phys. Chem. A 2006, 110 (18), 5990-5997.

7. DeRosa, M. C.; Crutchley, R. J. Photosensitized singlet oxygen and its applications. Coord. Chem. Rev. 2002, 233 (Supplement C), 351-371.

8. Ogilby, P. R. Singlet oxygen: there is indeed something new under the sun. Chem. Soc. Rev. 2010, $39(8), 3181-3209$. 
9. Cui, G. L.; Thiel, W. Intersystem Crossing Enables 4-Thiothymidine to Act as a Photosensitizer in Photodynamic Therapy: An Ab Initio QM/MM Study. J. Phys. Chem. Lett. 2014, 5 (15), 2682-2687.

10. Mai, S.; Pollum, M.; Martinez-Fernandez, L.; Dunn, N.; Marquetand, P.; Corral, I.; CrespoHernandez, C. E.; Gonzalez, L. The origin of efficient triplet state population in sulfur-substituted nucleobases. Nat. Commun. 2016, 7.

11. Brem, R.; Daehn, I.; Karran, P. Efficient DNA interstrand crosslinking by 6-thioguanine and UVA radiation. DNA Repair 2011, 10 (8), 869-876.

12. Zhang, X.; Jeffs, G.; Ren, X.; O’Donovan, P.; Montaner, B.; Perrett, C. M.; Karran, P.; Xu, Y.-Z. Novel DNA lesions generated by the interaction between therapeutic thiopurines and UVA light. DNA Repair 2007, 6 (3), 344-354.

13. Euvrard, S.; Kanitakis, J.; Claudy, A. Skin Cancers after Organ Transplantation. N. Engl. J. Med. 2003, 348 (17), 1681-1691.

14. Bai, S. M.; Barbatti, M. Why Replacing Different Oxygens of Thymine with Sulfur Causes Distinct Absorption and Intersystem Crossing. J. Phys. Chem. A 2016, 120 (32), 6342-6350.

15. Mai, S.; Marquetand, P.; González, L. Intersystem Crossing Pathways in the Noncanonical Nucleobase 2-Thiouracil: A Time-Dependent Picture. J. Phys. Chem. Lett. 2016, 7 (11), 1978-1983.

16. Schmidt, R. Quantitative determination of ${ }^{1} \Sigma_{\mathrm{g}}{ }^{+}$and ${ }^{1} \Delta_{\mathrm{g}}$ singlet oxygen in solvents very different polarity. General energy gap law for rate constants of electronic energy transfer to and from $\mathrm{O}_{2}$ in the absence of charge-transfer interactions. J. Phys. Chem. A 2006, 110 (34), 2622-2628.

17. Asturiol, D.; Barbatti, M. Electronic states of porphycene-O-2 complex and photoinduced singlet O-2 production. J. Chem. Phys. 2013, 139 (7).

18. Serrano-Perez, J. J.; Olaso-Gonzalez, G.; Merchan, M.; Serrano-Andres, L. Singlet oxygen generation in PUVA therapy studied using electronic structure calculations. Chem. Phys. 2009, 360 (13), 85-96.

19. Xie, B. B.; Liu, X. Y.; Fang, Q.; Fang, W. H.; Cui, G. L. The Position of the N Atom Plays a Significant Role for Excited-State Decay of Heterocycles. J. Phys. Chem. Lett. 2017, 8 (5), 1019-1024.

20. Martinez-Fernandez, L.; Gonzalez, L.; Corral, I. An ab initio mechanism for efficient population of triplet states in cytotoxic sulfur substituted DNA bases: the case of 6-thioguanine. Chem. Commun. 2012, 48 (15), 2134-2136. 
21. Mai, S.; Pollum, M.; Martínez-Fernández, L.; Dunn, N.; Marquetand, P.; Corral, I.; CrespoHernández, C. E.; González, L. The origin of efficient triplet state population in sulfur-substituted nucleobases. Nat. Commun. 2016, 7, 13077.

22. Boggio-Pasqua, M.; López Vidal, M.; Garavelli, M. Theoretical mechanistic study of selfsensitized photo-oxygenation and singlet oxygen thermal release in a dimethyldihydropyrene derivative. J. Photochem. Photobiol. A 2017, 333, 156-164.

23. Martínez-Fernández, L.; González-Vázquez, J.; González, L.; Corral, I. Time-Resolved Insight into the Photosensitized Generation of Singlet Oxygen in Endoperoxides. J. Chem. Theory Comput. 2015, $11(2), 406-414$.

24. Martínez-Fernández, L.; Corral, I.; Granucci, G.; Persico, M. Competing ultrafast intersystem crossing and internal conversion: a time resolved picture for the deactivation of 6-thioguanine. Chem. Sci. 2014, 5 (4), 1336-1347.

25. Chiodo, S. G.; Russo, N. DFT spin-orbit coupling between singlet and triplet excited states: A case of psoralen compounds. Chem. Phys. Lett. 2010, 490 (1-3), 90-96.

26. Bai, S.; Barbatti, M. On the decay of the triplet state of thionucleobases. Phys. Chem. Chem. Phys. 2017, 19 (20), 12674-12682.

27. Kuramochi, H.; Kobayashi, T.; Suzuki, T.; Ichimura, T. Excited-State Dynamics of 6-Aza-2thiothymine and 2-Thiothymine: Highly Efficient Intersystem Crossing and Singlet Oxygen Photosensitization. J. Phys. Chem. B 2010, 114 (26), 8782-8789.

28. Bai, S.; Barbatti, M. Divide to Conquer: A Kinetic Model for Singlet Oxygen Photosensitization. J. Chem. Theory Comput. 2017, doi:10.1021/acs.jctc.7b00619.

29. Berlin, Y. A.; Grozema, F. C.; Siebbeles, L. D. A.; Ratner, M. A. Charge transfer in donor-bridgeacceptor systems: static disorder, dynamic fluctuations, and complex kinetics. J. Phys. Chem. C 2008, 112 (29), 10988-11000.

30. Yeganeh, S.; Ratner, M. A.; Mujica, V. Dynamics of charge transfer: Rate processes formulated with nonequilibrium Green's functions. J. Chem. Phys. 2007, 126 (16), 161103.

31. Chen, H. N.; Ratner, M. A.; Schatz, G. C. Time-Dependent Theory of the Rate of Photo-induced Electron Transfer. J. Phys. Chem. C 2011, 115 (38), 18810-18821.

32. Harvey, J. N. Understanding the kinetics of spin-forbidden chemical reactions. Phys. Chem. Chem. Phys. 2007, 9 (3), 331-343. 
33. Harvey, J. N.; Aschi, M. Spin-forbidden dehydrogenation of methoxy cation: a statistical view. Phys. Chem. Chem. Phys. 1999, 1 (24), 5555-5563.

34. Banerjee, S.; Baiardi, A.; Bloino, J.; Barone, V. Temperature Dependence of Radiative and Nonradiative Rates from Time-Dependent Correlation Function Methods. J. Chem. Theory Comput. 2016, $12(2), 774-786$.

35. Chen, H.; Ratner, M. A.; Schatz, G. C. Time-Dependent Theory of the Rate of Photo-induced Electron Transfer. J. Phys. Chem. C 2011, 115 (38), 18810-18821.

36. Keszthelyi, T.; Poulsen, T. D.; Ogilby, P. R.; Mikkelsen, K. V. O-2(a(1)Delta(g)) absorption and O-2(b(1)Sigma(+)(g)) emission in solution: Quantifying the a-b Stokes shift. J. Phys. Chem. A 2000, 104 (45), 10550-10555.

37. Poulsen, T. D.; Ogilby, P. R.; Mikkelsen, K. V. The a(1)Delta(g) -> X-3 Sigma(-)(g) transition in molecular oxygen: Interpretation of solvent effects on spectral shifts. J. Phys. Chem. A 1999, 103 (18), 3418-3422.

38. Schweitzer, C.; Mehrdad, Z.; Noll, A.; Grabner, E. W.; Schmidt, R. Mechanism of photosensitized generation of singlet oxygen during oxygen quenching of triplet states and the general dependence of the rate constants and efficiencies of O-2((1)Sigma $(+)(g))$, O-2((1)Delta(g)), and O-2((3)Sigma(-)(g)) formation on sensitizer triplet state energy and oxidation potential. J. Phys. Chem. A 2003, 107 (13), $2192-$ 2198.

39. Abdel-Shafi, A. A.; Wilkinson, F. Electronic to vibrational energy conversion and charge transfer contributions during quenching by molecular oxygen of electronically excited triplet states. Phys. Chem. Chem. Phys. 2002, 4 (2), 248-254.

40. Jensen, P. G.; Arnbjerg, J.; Tolbod, L. P.; Toftegaard, R.; Ogilby, P. R. Influence of an Intermolecular Charge-Transfer State on Excited-State Relaxation Dynamics: Solvent Effect on the Methylnaphthalene-Oxygen System and its Significance for Singlet Oxygen Production. J. Phys. Chem. A 2009, 113 (37), 9965-9973.

41. Beljonne, D.; Yamagata, H.; Bredas, J. L.; Spano, F. C.; Olivier, Y. Charge-Transfer Excitations Steer the Davydov Splitting and Mediate Singlet Exciton Fission in Pentacene. Phys. Rev. Lett. 2013, 110 (22). 